\title{
QuAlidade de VIdA do IDOSO NA COMUNIdAdE: APLICAÇÃO DA ESCALA DE FLANAGAN
}

\author{
Sérgio Ribeiro dos Santos ${ }^{1}$ \\ Iolanda Beserra da Costa Santos ${ }^{1}$ \\ Maria das Graças M. Fernandes ${ }^{1}$ \\ Maria Emília Romero M. Henriques ${ }^{2}$
}

Santos SR, Santos IBC, Fernandes MGM, Henriques MERM. Qualidade de vida do idoso na comunidade: aplicação da escala de Flanagan. Rev Latino-am Enfermagem 2002 novembro-dezembro; 10(6):757-64.

Esse estudo objetivou avaliar a satisfação de idosos em relação à sua qualidade de vida. Os dados foram obtidos através de questionário que caracterizava a amostra e a Escala de Qualidade de Vida de Flanagan, utilizando-se a técnica de entrevista. A amostra foi constituída por 128 idosos. Nos resultados, verificamos que a satisfação desses idosos mostrou-se regular. As dimensões da qualidade de vida apontadas na escala de Flanagan foram: desenvolvimento pessoal e realização; relações com familiares; participação social; bem-estar físico e material; amizade e aprendizagem. Concluímos que essa Escala apresenta limitações em virtude do caráter subjetivo do conceito de qualidade.

DESCRITORES: qualidade de vida, saúde do idoso, nível de saúde

\section{ELDERLY QUALITY OF LIFE IN THE COMMUNITY: APPLICATION OF THE FLANAGAN'S SCALE}

The aim of this study was to evaluate the satisfaction degree of the elderly people regarding their quality of life. Data were obtained by a questionnaire characterizing the sample with a scale of quality proposed by Flanagan, using the interview technique. Among 128 elderly people, authors verified a insufficient degree of satisfaction. The dimensions of the quality of life pointed out by the Flanagan's scale were: personal development and fulfillment; family relations; social participation; welfare and material support; learning and friendship. The authors concluded that the Flanagan's scale presents limitations as a result of the subjectivity of the concept of quality.

DESCRIPTORS: quality of life, elderly health, health status

\section{CALIdAd dE VIdA del ANGIANO EN LA COMUNIdAd: APLICACIÓN de LA ESCALA DE FLANAGAN}

Este estudio pretendió valorar la satisfacción de los ancianos en relación a su calidad de vida. Los datos fueron obtenidos por medio de un cuestionario que caracterizaba la muestra y la escala de calidad de vida de Flanagan, utilizando la técnica de entrevista. La muestra fue constituida por 128 ancianos. En los resultados, comprobamos que la satisfacción de estos ancianos fue regular. Las dimensiones de la calidad de vida apuntadas en escala de Flanagan fueron: desarrollo personal y realización, relaciones con familiares, participación social, bienestar físico y material, amistad y aprendizaje. Concluimos que esta escala presenta limitaciones en virtud del carácter subjetivo del concepto de calidad.

DESCRIPTORES: calidad de vida, salud del anciano, estado de salud

\footnotetext{
${ }_{1}^{1}$ Enfermeiros, Mestres em Enfermagem, Doutorandos em Ciências da Saúde/Universidade Federal da Paraíba, e-mail: s.r.santos@uol.com.br; 2 Enfermeira, Doutor em Enfermagem, Professor do Programa de Pós-Graduação em Ciências da Saúde/Universidade Federal da Paraíba, Chefe do Departamento de Enfermagem-DEMCA/CCS/Universidade Federal da Paraíba
} 


\section{INTRODUÇÃO}

A Qualidade de Vida - QV tem sido preocupação constante do ser humano, desde o início de sua existência e, atualmente, constitui um compromisso pessoal a busca contínua de uma vida saudável, desenvolvida à luz de um bem-estar indissociável das condições do modo de viver, como: saúde, moradia, educação, lazer, transporte, liberdade, trabalho, auto-estima, entre outras.

O termo Qualidade de Vida tem recebido uma variedade de definições ao longo dos anos. A QV pode se basear em três princípios fundamentais: capacidade funcional, nível sócioeconômico e satisfação ${ }^{(1)}$. A QV também pode estar relacionada com os seguintes componentes: capacidade física, estado emocional, interação social, atividade intelectual, situação econômica e autoproteção de saúde ${ }^{(2)}$. Na realidade, o conceito de QV varia de acordo com a visão de cada indivíduo. Para alguns, ela é considerada como unidimensional, enquanto, para outros, é conceituada como multidimensional ${ }^{(3)}$.

A QV boa ou excelente é aquela que oferece um mínimo de condições para que os indivíduos possam desenvolver o máximo de suas potencialidades, vivendo, sentindo ou amando, trabalhando, produzindo bens ou serviços; fazendo ciência ou artes; vivendo (...) apenas enfeitando, ou, simplesmente existindo. Todos são seres vivos que procuram se realizar ${ }^{(4)}$. Por outro lado, muitas pessoas procuram associar qualidade de vida com o fator saúde. Nesse sentido, saúde, independente de qualquer definição idealista que Ihe possa ser atribuída, é produto das condições objetivas de existência. Resulta das condições de vida biológica, social e cultural e, particularmente, das relações que os homens estabelecem entre si e com a natureza, através do trabalho ${ }^{(5)}$.

Assim, como é difícil conceituar Qualidade de Vida, a sua medida também o é, já que ela pode sofrer influência de valores culturais, éticos e religiosos, bem como de valores e percepções pessoais ${ }^{(6)}$. Esse caráter de subjetividade de que se reveste o conceito de qualidade de vida baseia-se na realidade individual que converge no subjetivismo, constituindo-se, assim, num conceito muito complexo e de difícil avaliação ${ }^{(2,7)}$.

Considerando a multidimensionalidade, assim como os vários significados associados à definição de qualidade de vida, observados no âmbito das diversas disciplinas, Flanagan propôs uma escala para avaliar tal conceito que é bastante utilizada nos Estados Unidos da
América, pela validade e confiabilidade de seus achados, que contempla o grau de satisfação individual ou percebido com relação a cinco dimensões da vida: bem-estar físico e material; relacionamentos; atividades sociais, comunitárias e cívicas; desenvolvimento e realização pessoal e recreação ${ }^{(8)}$.

Numa aproximação entre a concepção de qualidade de vida proposta por Flanagan em sua escala com a literatura pertinente, observa-se, quanto aos conceitos inter-relacionados expressos em suas dimensões e itens como: bem-estar "refere-se à boa disposição física, conforto, tranqüilidade"(9); relacionamento, compreende "ligação afetiva, condicionada por uma série de atitudes recíprocas" ${ }^{\prime 9)}$; atividade, é definida como comportamentos emitidos como resposta a eventos ${ }^{(10)}$, que atuariam sobre a competência funcional do indivíduo. Autodesenvolvimento, consiste na autoaceitação, relações positivas com outros, autonomia, senso de domínio, busca de metas ${ }^{(10)}$. Recreação, compreende as atividades de distração de significado individual e social ${ }^{(11)}$.

No tocante à avaliação da qualidade de vida do idoso, deve-se ressaltar a complexidade da tarefa e, como tal, reforçar a importância da utilização de critérios de avaliação híbridos, como os contemplados na escala de Flanagan, que permitam clarificar aspectos intersubjetivos que têm maior probabilidade de ocorrência em idosos do que em adultos jovens, tais como: doenças, perdas de papéis ocupacionais e perdas afetivas.

Avaliar a qualidade de vida do idoso implica a adoção de múltiplos critérios de natureza biológica, psicológica e socioestrutural, pois vários elementos são apontados como determinantes ou indicadores de bemestar na velhice: longevidade, saúde biológica, saúde mental, satisfação, controle cognitivo, competência social, produtividade, atividade, eficácia cognitiva, status social, renda, continuidade de papéis familiares, ocupacionais e continuidade de relações informais com amigos ${ }^{(10)}$.

Ante o exposto, avaliar as condições de vida do idoso reveste-se de grande importância científica e social por permitir a implementação de alternativas válidas de intervenção, tanto em programas gerontogeriátricos, quanto em políticas sociais gerais, no intuito de promover o bemestar das pessoas maduras, particularmente, no nosso contexto, onde os atuais idosos são aqueles que conseguiram sobreviver às condições adversas ${ }^{(12)}$.

Essas situações determinam as implicações com 
relação às potencialidades de saúde e de vida do idoso, interferindo no seu processo saúde-doença. Diante do exposto, esse estudo procurou resposta para o seguinte questionamento: que avaliação os idosos fazem de sua qualidade de vida no contexto comunitário? Para encontrar resposta a essa questão, definiu-se o seguinte objetivo: avaliar a satisfação do idoso em relação a sua qualidade de vida.

\section{METODOLOGIA}

O método utilizado neste estudo foi de natureza quantitativa. A população foi constituída por cinco grupos de idosos em diferentes comunidades da cidade de João Pessoa - Paraíba. Para composição da amostra, optouse pela amostragem por acessibilidade por ser esse um procedimento que é destituído de qualquer rigor estatístico. $\mathrm{Na}$ seleção da amostra foram considerados indivíduos idosos aqueles que estavam na faixa etária de 60 anos ou mais. Esse critério é adotado pela Organização Mundial de Saúde para definir pessoa idosa, do ponto de vista cronológico, nos países em desenvolvimento, a exemplo do Brasil ${ }^{(13)}$

Para a coleta dos dados, utilizou-se uma entrevista estruturada composta de duas partes: a primeira, constituindo-se dos dados sóciodemográficos e, a segunda, da Escala de Qualidade de Vida de Flanagan $^{(14)}$, que conceptualiza qualidade de vida a partir de cinco dimensões, bem-estar físico e material, relações com outras pessoas, atividades sociais, comunitárias e cívicas, desenvolvimento pessoal e realização, e recreação. Essas dimensões são mensuradas através de quinze itens onde o respondente tem sete opções de resposta, que vai de "muito insatisfeito" (escore 1) até "muito satisfeito" (escore 7). A pontuação máxima alcançada na avaliação da qualidade de vida proposta por Flanagan ${ }^{(14)}$ é de 105 pontos e a mínima de 15 pontos, que refletem baixa qualidade de vida. Cabe destacar que a escala é auto-aplicável, no entanto, alguns idosos envolvidos neste estudo receberam auxílio dos pesquisadores para responderem tal instrumento, pois apresentavam limitações físicas como tremores das mãos, diminuição da acuidade visual e auditiva, e baixo nível de escolaridade.

A Escala de Qualidade de Vida de Flanagan EQVF-foi desenvolvida no âmbito dos Estados Unidos. Apesar de não ter sido validada na cultura brasileira,
Hashimoto e colaboradores realizaram sua tradução para o português e aplicaram em pacientes ostomizados ${ }^{(8)}$. Em 1998, Gonçalves et al. aplicaram a escala numa amostra aleatória relativamente extensa e heterogênea e observaram alta confiabilidade do instrumento. Em seguida, utilizou tal escala numa pesquisa envolvendo idosos ${ }^{(8)}$, verificando um bom nível de confiabilidade, aspecto que contribuiu para a decisão do uso do referido instrumento nesta pesquisa.

Ressaltamos que em todo o processo da pesquisa, especialmente durante a coleta de dados, obedecemos aos princípios éticos dispostos na Resolução No 196/96, do Conselho Nacional de Saúde ${ }^{(15)}$, especificamente, no seu artigo IV, que aborda o respeito à autonomia do participante da pesquisa, garantindo-lhe, entre outros direitos, o seu consentimento livre e esclarecido, o sigilo das informações e a privacidade. Esclarecemos que a coleta de dados teve início após aprovação do projeto de pesquisa na Comissão de Bioética do Centro de Ciências da Saúde - UFPB.

Os dados foram agrupados em estratos e tratados estatisticamente, utilizando-se sistema computacional, através do programa Statistical Package for the Social Sciences - SPSS, que permite manusear vários tipos de análise de dados de maneira simples e conveniente, oferecendo grande número de rotinas estatísticas, das quais utilizamos a estatística descritiva e da análise fatorial.

\section{RESULTADOS E DISCUSSÃO}

O presente estudo foi desenvolvido com cinco grupos de idosos das comunidades de Cruz das Armas, Bairro dos Estados, Bairro dos Ipês, Róger e Jardim Luna, no município de João Pessoa-PB, no período de 25 de julho a 04 de agosto de 2000, totalizando 128 idosos com idade mínima de 60 anos e máxima de 91 anos. A idade média do grupo pesquisado foi 70 anos, que está próximo da expectativa média de vida do brasileiro, na atualidade.

\section{Dados sóciodemográficos}

No tocante aos dados sóciodemográficos pertinentes aos participantes do estudo, foram investigados o sexo, o estado civil, a religião, a moradia, convivência, ocupação e renda. Pode-se observar, na Tabela 1, que há predominância do sexo feminino $(79,7 \%)$, seguido do sexo 
masculino $(20,03 \%)$. Tais resultados confirmam a tendência nos estudos relacionados com os idosos ${ }^{(8,16)}$, ao verificarem que nos grupos de idosos a participação masculina raramente ultrapassa $20 \%$, de forma que imaginamos ser a velhice, no Brasil, uma experiência essencialmente feminina.

É importante ressaltar que o número absoluto de mulheres idosas tem sido superior, no Brasil, quando confrontado com o de homens de 65 anos ou mais. Essa situação decorre da existência de mortalidade diferencial por sexo que prevalece há muito tempo na população brasileira ${ }^{(17)}$. Desde 1950, as mulheres possuem maior esperança de vida. Em 1980, enquanto a expectativa de vida para os homens era de 59 anos, para as mulheres era de 65 anos. Em 1991, essa diferença cresceu para sete anos.

Tabela 1 - Distribuição das características apresentadas pelos idosos de grupos comunitários da cidade de João PessoaPB, 2000

\begin{tabular}{|c|c|c|c|c|c|}
\hline \multirow[b]{2}{*}{ CARACTERÍSTICAS } & \multicolumn{2}{|c|}{ PARTICIPANTES } & \multirow[b]{2}{*}{ CARACTERÍSTICAS } & \multicolumn{2}{|c|}{ PARTICIPANTES } \\
\hline & $\mathrm{N}^{\circ}$ & $\%$ & & $\mathrm{~N}^{\mathrm{o}}$ & $\%$ \\
\hline SEXO & & & OCUPAÇÃO & & \\
\hline Feminino & 102 & 79,7 & Aposentado & 60 & 46,9 \\
\hline Masculino & 26 & 20,3 & Doméstica & 53 & 41,4 \\
\hline ESTADO CIVIL & & & Outros & 15 & 11,7 \\
\hline Viúvo & 53 & 41,4 & RENDA & & \\
\hline Casado & 51 & 39,8 & Menos de 1 salário mínimo & 16 & 12,5 \\
\hline Solteiro & 16 & 12,5 & De 1 até 3 salários mínimo & 97 & 75,8 \\
\hline Separado & 8 & 6,3 & De 3 até 5 salários mínimo & 7 & 5,5 \\
\hline RELIGIÃO & & & Acima de 5 salários mínimo & 8 & 6,3 \\
\hline Católico & 112 & 87,5 & MORADIA & & \\
\hline Evangélico & 12 & 9,4 & Própria & 105 & 82,0 \\
\hline Outras & 4 & 3,2 & Alugada & 11 & 8,6 \\
\hline CONVIVÊNCIA & & & Dos filhos & 8 & 6,3 \\
\hline Outros familiares & 71 & 55,5 & Outros & 4 & 3,1 \\
\hline Cônjuge & 35 & 27,3 & & & \\
\hline Só & 19 & 14,8 & & & \\
\hline Outros & 3 & 2,3 & & & \\
\hline
\end{tabular}

Com relação ao estado civil, os viúvos predominaram nessa amostra, com $41,4 \%$, seguido pelos casados, com $39,8 \%$. Considerando que as mulheres idosas constituem o maior número de participantes desse estudo $(79,7 \%)$, aplica-se à interpretação desse dado a analogia feita por Chamowicz quanto à situação conjugal dos idosos brasileiros:

"Em 1993, a grande maioria das mulheres idosas (66\%) era composta por viúvas, solteiras ou separadas, um enorme contraste com a situação dos homens de mesma idade ( $76,3 \%$ casados). Tais diferenças se explicam, não somente pela menor longevidade dos homens, mas, também, pela maior freqüência de recasamento dos homens, após a viuvez, e maior tendência destes se casarem com mulheres mais jovens"(18).

$O$ aspecto religioso tem grande influência nessa fase da vida, de forma que $100 \%$ da população pesquisada (128) demonstraram afinidade com algum tipo de atividade ou prática religiosa, predominando o engajamento na religião católica, com $87,5 \%$, seguida da religião evangélica, com 9,4\%. Quanto às razões para a ocorrência desse fato, verifica-se que a prática de uma religião pelo idoso permite-lhe estabelecer um elo entre as limitações e o aproveitamento de suas potencialidades ou, quando 
isso não ocorre, ajuda-o a vencer com mais facilidade essa última etapa da vida ${ }^{(19)}$. A moradia e a convivência têm sido a grande preocupação dos idosos, porém, nessa amostra, constatou-se que $82 \%$ deles possuem casa própria, significando que a maioria acumulou, ao longo dos anos, algum patrimônio. Por outro lado, 55,5\% convivem com outros familiares em seu domicílio e apenas $27,3 \%$ vivem com seus cônjuges. Porém, um dado que nos surpreendeu foi a quantidade de idosos que vivem sozinhos (14,8\%), resultado esse também identificado em outros estudos $^{(8,16)}$.

A situação familiar dos idosos reflete o efeito acumulado de eventos sócioeconômico e demográficos e de saúde ocorridos em etapas anteriores do ciclo vital. 0 tamanho da prole, a mortalidade diferencial, o celibato, a viuvez, as separações, os recasamentos e as migrações vão originando, ao longo do tempo, distintos tipos de arranjos familiares e domésticos. Morar sozinho, ou morar com parentes pode ser o resultado desses mesmos desenlaces $^{(17)}$.

É importante destacar que há idosos que permanecem desenvolvendo alguma atividade, mesmo após a aposentadoria, como forma de complementar o orçamento doméstico. Verificou-se, no grupo estudado, que $41,4 \%$ exercem atividades domésticas; $3,1 \%$ são costureiras e $46,9 \%$ aposentados. Os demais trabalham como lavadeira, professor, vendedor, vigia e outros. Percebe-se, pela condição de trabalho e posição social, que a renda dessa amostra é pequena, predominando em $75,8 \%$ a renda de 1 até 3 salários mínimos e em 12,5\% menos de 1 (um) salário-mínimo.

Uma das características marcantes da população idosa no Brasil é seu baixo poder aquisitivo. Aposentadorias e pensões constituem sua principal fonte de rendimentos. Como conseqüência do baixo valor dos benefícios, um terço dos brasileiros com 60 anos ou mais se mantinham em atividades produtivas em 1995. O retorno dos idosos ao mercado de trabalho ou a sua permanência aí se dá sobretudo no mercado informal, em atividades mal remuneradas e jornadas de trabalho extensas. Em 1998, a maioria dos idosos integrados à população economicamente ativa possuía renda proveniente do trabalho inferior ou igual a dois salários-mínimos e exerciam atividades pouco compatíveis com a idade, trazendo-lhes desgaste físico cada vez mais acentuado ${ }^{(18)}$.

Quando indagados sobre sua maior preocupação no momento, obtivemos como resposta predominante do grupo o estado de saúde (25,8\%). Esse resultado, de algum modo, era esperado, pois no contexto do envelhecimento o ser humano apresenta maior fragilidade do seu organismo, podendo favorecer o surgimento de doenças e outros desconfortos físicos, psíquicos e sociais. Além dessa, outras preocupações foram evidenciadas: como a família $(15,6 \%)$, por razões como "filho desempregado, alcoólatra, desajustado financeiramente, violento, viciado em drogas, separação conjugal"; com a renda $(8,6 \%)$, por não ser suficiente para atender às suas necessidades básicas; com a casa $(7 \%)$, carecendo reformas; e, por fim, com a solidão (3,9\%), por não terem como quem compartilhar seus anseios, desejos e experiências.

Qualidade de vida dos idosos através da aplicação da Escala de Flanagan

A Escala de Qualidade de Vida de Flanagan EQVF - foi aplicada ao grupo de idosos que avaliaram a sua satisfação em relação a 15 itens agrupados em 05 fatores, como mostrado na Tabela 2. A média dos escores obtidos, nessa amostra de idosos, foi de 74,32 e o coeficiente de confiabilidade alpha igual a 0,7635 para 128 casos investigados. Esses resultados confirmam a eficiência do instrumento, pois um valor para alpha maior que 0,6000 significa que houve concordância entre o instrumento e a população alvo.

O teste de significância de correlação de matrizes para análise fatorial Bartlett's teste de esfericidade e a medida Kaiser-Meyer-Olkin (KMO) também foram identificados nessa amostra. A medida de $\mathrm{KMO}$ para o grupo de idosos foi igual a 0,711. Quando a KMO se aproxima do valor um, há suporte para o método de análise fatorial. O teste de Bartlett's foi igual a 408,26, o que significa que houve correlação entre os itens da escala de Flanagan.

Essas informações possibilitaram a utilização da análise dos componentes principais, através do programa SPSS/PC ${ }^{(20)}$. Isso nos permitiu confrontar os resultados dessa amostra com os dimensionamentos expostos na escala de Flanagan, de forma que aplicamos o método de rotação varimax sobre os itens da escala. Somente fatores com eigenvalue maiores que 1 foram aceitos e apenas os itens com carga fatorial acima de 0,5000 foram considerados para efeito de identificação dos componentes principais, como demonstra a Tabela 2. 
Dos 15 (quinze) itens contidos na escala de Flanagan, aplicados nessa amostra, apenas os itens $7 \mathrm{e}$ 14 foram excluídos porque não apresentaram carga fatorial dentro do limite estabelecido para esse estudo $(0,5000)$.

Tabela 2 - Demonstrativo da aplicação da análise fatorial e identificação dos componentes principais que influenciaram no nível de satisfação com a qualidade de vida da amostra de idosos na cidade de João Pessoa-PB, 2000

\begin{tabular}{|c|c|}
\hline ENUNCIADOS DA ESCALA FLANAGAN & $\begin{array}{l}\text { Carga } \\
\text { Fatorial }\end{array}$ \\
\hline $\begin{array}{l}\text { FATOR } 1 \text { - Desenvolvimento pessoal e realização } \\
\text { (Variância } 24,56 \% \text { e Eigenvalue } 3,685 \text { ) }\end{array}$ & \\
\hline $\begin{array}{l}\text { Item } 10 \text { - Autoconhecimento: reconhecer seus } \\
\text { potenciais e limitações. }\end{array}$ & 0,806 \\
\hline $\begin{array}{l}\text { Item } 11 \text { - Trabalho(emprego ou em casa): atividade } \\
\text { interessante. }\end{array}$ & 0,680 \\
\hline $\begin{array}{l}\text { Item } 12 \text { - Comunicação criativa } \\
\text { FATOR } 2 \text { - Relações com familiares (Variância } \\
10.88 \% \text { e Eigenvalue } 1.633 \text { ) }\end{array}$ & 0,662 \\
\hline $\begin{array}{l}\text { Item } 3 \text { - Relacionamento com pais, irmãos e outros } \\
\text { parentes. }\end{array}$ & 0,709 \\
\hline a: ter e criar filhos. & +5 \\
\hline $\begin{array}{l}\text { Item } 5 \text { - Relacionamento íntimo com esposo(a), } \\
\text { namorado(a) ou outra pessoa. } \\
\text { FATOR } 3 \text { - Participação social (Variância } 9,17 \% \text { e } \\
\text { Eigenvalue } 1,376 \text { ) }\end{array}$ & 89 \\
\hline $\begin{array}{l}\text { Item } 8 \text { - Participação em associações e atividades } \\
\text { de interesse público. }\end{array}$ & 0,765 \\
\hline Item $13-\mathrm{Pa}$ & 0,763 \\
\hline $\begin{array}{l}\text { Item } 15 \text { - Socialização: fazer amizades } \\
\text { FATOR } 4 \text { - Bem-estar físico e material (Variância } \\
8,04 \% \text { e Eigenvalue } 1,207 \text { ) }\end{array}$ & 0,601 \\
\hline $\begin{array}{l}\text { Item } 1 \text { - Conforto material: casa, alimentação, } \\
\text { situação financeira. }\end{array}$ & 0,787 \\
\hline $\begin{array}{l}\text { Item } 2 \text { - Saúde: fisicamente bem e vigoroso(a). } \\
\text { FATOR } 5 \text { - Amizade e aprendizagem (Variância } \\
6,39 \% \text { e Eigenvalue } 1,041 \text { ) }\end{array}$ & 0,84 \\
\hline $\begin{array}{l}\text { Item } 6 \text { - Amigos próximos: compartilhar interesses, } \\
\text { atividades e opiniões }\end{array}$ &, 791 \\
\hline $\begin{array}{l}\text { Item } 9 \text { - Aprendizagem: freqüentar cursos para } \\
\text { conhecimentos gerais }\end{array}$ & 0,50 \\
\hline
\end{tabular}

Os resultados demonstraram algumas divergências no tocante às dimensões do conceito de qualidade de vida propostas por Flanagan ${ }^{(14)}$ e aquelas consideradas pelos idosos como de maior importância na determinação de sua qualidade de vida, como pode ser demonstrado na Tabela 3. Tal fato sugere que a EQVF pode sofrer variações dependendo da população estudada e do contexto no qual essa população está inserida. Os idosos investigados evidenciaram peculiaridades e expectativas próprias de forma que a ordem de prioridade que eles concebem a qualidade de vida é diferente daquela apresentada por outros indivíduos, inclusive da mesma faixa etária, participantes de outros estudos onde foi utilizado o mesmo instrumento, a exemplo do desenvolvido por Gonçalves ${ }^{(8)}$. Por essa razão, os itens 7 e 14 exerceram pouca influência na qualidade de vida dessa amostra.
Tabela 3 - Demonstrativo de comparação das dimensões da EQVF e as identificadas na amostra de 128 idosos na cidade de João Pessoa, 2000

\begin{tabular}{ll}
\hline \multicolumn{1}{c}{ DIMENSÕES DA EQVF } & $\begin{array}{c}\text { DIMENSÕES IDENTIFICADAS } \\
\text { NA AMOSTRA }\end{array}$ \\
\hline \begin{tabular}{ll} 
1. Bem-estar físico e material & $\begin{array}{l}\text { 1. Desenvolvimento pessoal e } \\
\text { realização }\end{array}$ \\
2. Relações com outras pes- & 2. Relações com familiares \\
soas & \\
$\begin{array}{l}\text { 3. Atividades sociais, comunitá- } \\
\text { rias e cívicas }\end{array}$ & 3. Participação social \\
4. Desenvolvimento pessoal e & 4. Bem-estar físico e material \\
realização & \\
5. Recreação & 5. Amizade e aprendizagem \\
\hline
\end{tabular}
\end{tabular}

Para chegarmos a esses resultados e termos melhor compreensão da análise fatorial, vários testes foram realizados, aplicando-se o método de rotação varimax, procurando-se encontrar o agrupamento de variáveis que representassem, com mais clareza, o nível de satisfação dos idosos com a sua qualidade de vida, isso porque a maior dificuldade no uso desse método é a interpretação dos resultados, de acordo com a realidade da amostra. Isso significa que podemos obter uma resposta diferente, se aplicarmos o mesmo instrumento em outra população. Assim sendo, podemos verificar que a extração de cinco fatores ou dimensões para essa amostra de idosos reflete a melhor interpretação.

Esses dados revelam a existência de qualidade de vida regular nas diversas variáveis analisadas pelos respondentes. É importante destacar que, dos quinze itens da escala, os idosos demonstraram baixa satisfação para os escores menores que 5. Assim, pode-se verificar na Figura 1, que 8 itens enquadram-se nesse critério. É interessante observar que a variável 15 - Socialização: "fazer amigos", apresentou maior escore de satisfação, com média de 6,23, alinhando-se perfeitamente com o estado atual de satisfação. Esse escore reflete as atividades exercidas nos grupos comunitários com envolvimento nas tarefas sociais e religiosas que facilitam a interação com novas amizades, permitindo-lhes novas possibilidades de socialização e de trocas de experiências num território que pode simbolizar o público ou o privado, a rua ou a casa, ou, ainda, o salão de festas de antigamente. 


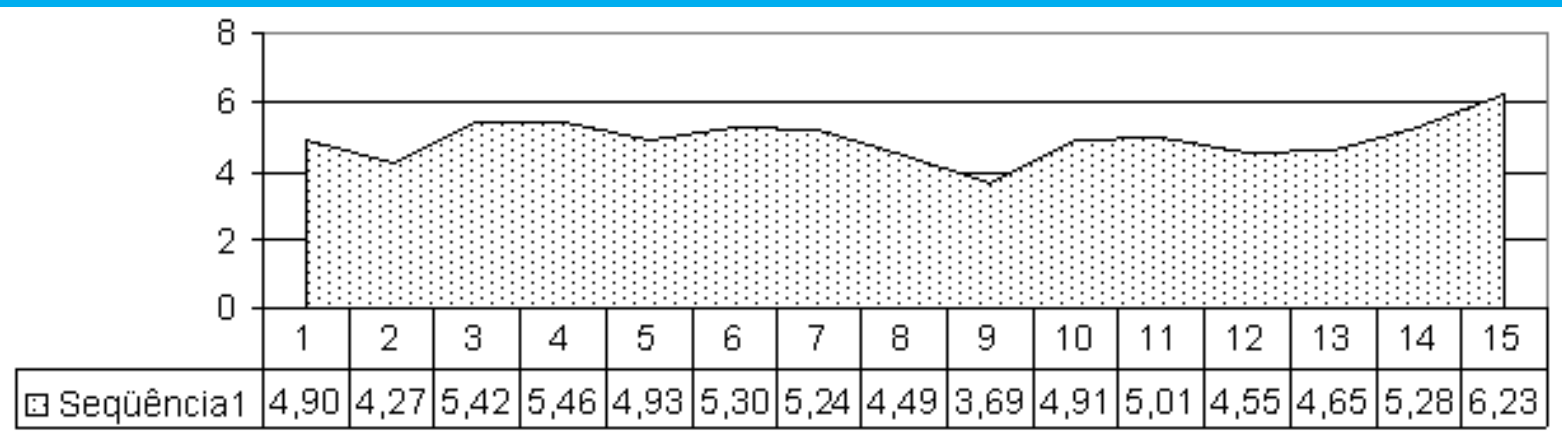

LEGENDA: 1 - Conforto material; 2 - Saúde; 3 - Relação com parentes; 4 - Constituir família; 5 - Relacionamento íntimo; 6 - Amigos; 7 - Ajuda; 8 - Participação em associações; 9 - Aprendizagem; 10 - Autoconhecimento; 11 - Trabalho; 12 - Comunicação; 13 - Recreação ativa; 14 - Ouvir música; 15 - Socialização

Figura 1 - Gráfico da média de freqüência obtida nos 15 itens da EQV de Flanagan na amostra de idosos da cidade de João Pessoa-PB, 2000

As relações familiares verificadas como dimensão importante na vida dos participantes do estudo constituiu um dos fatores que gerou prejuízo na sua qualidade de vida. O envelhecimento é uma importante questão familiar, independentemente da vivência conjunta ou não das gerações, dada à especificidade do relacionamento nessa etapa do ciclo vital, em decorrência, principalmente, da perda de papéis por parte do senescente, bem como da transferência desses papéis para os filhos, demandando transformações nas maneiras de definir os elementos presentes na experiência familiar ${ }^{(21)}$. Entre os eventos desencadeadores de crises nas relações familiares do grupo investigado destacamos a independência dos filhos, gerando o "ninho vazio"; o não atendimento das necessidades básicas da família, em conseqüência do baixo valor da sua aposentadoria; e os conflitos intergeracionais. Frente a essa realidade, as relações familiares dos idosos influenciaram e foram influenciadas por outros aspectos ou dimensões da qualidade de vida.

No geral, observamos que a qualidade de vida da amostra de idosos, numa graduação, vai de pouca a moderada satisfação (a Figura 1 sintetiza esse perfil, exibindo a média geral de atitudes da amostra de idosos). Esse nível de satisfação com a qualidade de vida, de pouco a moderado, evidenciado pelos idosos em 13 itens da Escala de Flanagan, resulta da condição biopsicossocial experimentada por esses indivíduos no contexto brasileiro. Assim, "como esperar do idoso satisfação com sua vida quando o que a sociedade the oferece são tribulações e agressões econômicas, sociais e ambientais? Sem falar de assistência médica não assegurada, poluição sonora e visual, circulação ameaçadora nas ruas das cidades, além de outras emoções negativas resultantes de um mundo em aceleradas transformações que exclui os idosos de seu meio"(22). Nesse contexto, "estar bem com a vida" não passa de uma idealidade imaginária.

\section{CONSIDERAÇÕES FINAIS}

Nesse estudo verificamos que a Escala de Flanagan apresenta resultado variado dependendo da população investigada, conforme foi constatado através da análise fatorial, e nos conduziu à extração de componentes principais sobre os itens propostos pela escala. Para essa amostra foram extraídos 05 (cinco) fatores ou dimensões que se inter-relacionam. Porém, na ordem prioritária de satisfação, temos: desenvolvimento pessoal e realização; relações com familiares; participação social; bem-estar físico e material; amizade e aprendizagem.

Também foram identificados algumas limitações em alguns itens da EQVF que, conforme nosso entendimento, são pouco operacionais, pois não evidenciam, plenamente, comportamentos físicos por meio dos quais o constructo de qualidade de vida se expressa, como, por exemplo, comunicação criativa, recreação ativa, aprendizagem, resultando em dificuldades quanto à sua compreensão pelos idosos pesquisados. Considerando as particularidades dos idosos que implicaram em algumas limitações na aplicabilidade da escala, destacam-se os atributos do processo de envelhecimento nas suas múltiplas dimensões, bem como a relação dos senescentes com a sociedade e a família que, no geral, suscita nos idosos padrões de comportamentos que prejudicam sua qualidade de vida. Considerando o item 
"saúde" é comum os idosos manifestarem alterações da saúde, inclusive pluripatologias multideterminadas, tornado sua satisfação com esse item pouco observada.

Assim, concluímos que a Escala de Flanagan, como instrumento de avaliação da qualidade de vida,

\section{REFERÊNCIAS BIBLIOGRÁFICAS}

1. González NM. Symposium de calidad de vida: generalidades, mediciones utilizadas en medicina, elementos que la componen. Arch Reumatol 1993; 4(1):402.

2. Hornquist JO. Quality of life: concept and assessment. Scand J Soc Med 1990; 18:69-79.

3. Zhan L. Quality of life: conceptual and measurement issues. J Adv Nurs 1992; 17(7):795-800.

4. Ruffino AN. Qualidade de vida: compromisso histórico da epidemiologia. Saúde em Debate, 1992; 35:63-7.

5. Paim JS. Direito à saúde, cidadania e estado. Anais da $8^{a}$ Conferência Nacional de Saúde; 1986; Brasília: Ministério da Saúde; 1986. p.45-59.

6. Gaíva MAM. Qualidade de vida e saúde. Rev Enf UERJ 1998 dezembro; 6(2):377-82.

7. Segre M, Ferraz FC. O conceito de saúde. Rev Saúde Pública 1997; 31(5):538-42.

8. Gonçalves LHT, Dias MM, Liz TG. Qualidade de vida de idosos independentes segundo proposta de avaliação de Flanagan. O mundo da Saúde 1999 jul/ago; 23(4):214-20.

9. Larousse Cultural. Enciclopédia Larousse Cultural. São Paulo (SP): Nova Cultura; 1992.

10. Neri AL. Qualidade de vida e idade madura. Campinas (SP): Papirus; 1993.

11. Moragas RM. Gerontologia social: envelhecimento e qualidade de vida. São Paulo (SP): Paulinas; 1997.

12. Veras RP. País jovem com cabelos brancos: a saúde do idoso no Brasil. Rio de Janeiro (RJ): Relume Dumará - UERJ; 1994.

13. Ministério da Previdência e Assistência Social. Secretaria de Ação Social (BR). Plano de ação governamental integrado para o desenvolvimento da Política Nacional do Idoso. Brasília (DF): Ministério da Previdência e Assistência Social. Secretaria de Ação Social; 1996.

14. Flangan, JC. Measurement of quality of life: currente of art state. Arch. Phys. Med. Rehabil. 1982; 23:56-59.

15. Ministério da Saúde (BR). Conselho Nacional de Saúde. Comissão Nacional de Ética em Pesquisa - CONEP. Resolução n 196/96. Dispõe sobre pesquisa envolvendo seres humanos. Brasília (DF): Ministério da Saúde; 1996.

16. Derbert GG. As representações (esteriótipos) do perfil do idoso na sociedade atual. Anais do I Seminário Internacional Envelhecimento Populacional: uma agenda para o final do século; Brasília (BR): MPAS/SAS; 1996. p. 35-45.

17. Berquó E. Algumas considerações demográficas sobre o envelhecimento da população no Brasil. In: Anais do I Seminário Internacional Envelhecimento Populacional: uma agenda para o final do século. Brasília (BR): MPAS/SAS; 1996. p. 16-34.

18. Chamowicz F. Os idosos brasileiros no século XXI: demografia, saúde e sociedade. Belo Horizonte (MG): PostGraduate; 1998.

Recebido em: 29.9.2000

Aprovado em: 30.6.2002 apresentou limitações, porém precisa de adequação à realidade objeto da pesquisa, em virtude do caráter subjetivo do conceito de qualidade de vida e do entendimento do idoso sobre o modelo operacional de aplicação do instrumento.

19. Araújo CDSF. Aspectos religiosos do idoso. In: Petroiano A, Pimenta LG. Clínica e cirurgia geriátrica. Rio de Janeiro (RJ): Guanabara; 1999. p. 8-9.

20. Norusis MJ. SPSS/PC. Professional statistics. Chicago: SPSS Inc.; 1992.

21. Ângelo M. O contexto familiar. In.: Duarte YAO, Diogo MID. Atendimento domiciliar: um enfoque gerontológico. São Paulo (SP): Atheneu; 2000. p. 27-31.

22. Corrêa JA. O presente da velhice: reflexão filosófica sobre a velhice. In: Petroiano A, Pimenta LG. Clínica e cirurgia geriátrica. Rio de Janeiro (RJ): Guanabara; 1999. p. 1-7. 